\title{
Research Article \\ High-Precision Extraction Method for Blade Tip-Timing Signal with Eddy Current Sensor
}

\author{
Ji-wang Zhang $\mathbb{D}^{\mathrm{D}}$, Ke-qin Ding, and Guang Chen \\ China Special Equipment Inspection and Research Institute, Beijing 100029, China \\ Correspondence should be addressed to Ji-wang Zhang; zhangjiwang@csei.org.cn
}

Received 17 June 2020; Accepted 26 October 2020; Published 16 November 2020

Academic Editor: Funazaki Ken-ichi

Copyright ( 2020 Zhang Ji-wang et al. This is an open access article distributed under the Creative Commons Attribution License, which permits unrestricted use, distribution, and reproduction in any medium, provided the original work is properly cited.

\begin{abstract}
Online monitoring of high-speed rotating blades has always been a hot topic. Of the various methods, the blade tip timing (BTT) technique, based on eddy current sensors, is considered to be the most promising. However, BTT signals are easily influenced by various factors, which means that the accurate extraction of BTT signals remains a challenge. To try to solve this problem, the causes of measurement error were analyzed. The three main reasons for the error were established: the variation in blade tip clearance, the interference of background noise, and the asymmetry of the blade tip shape. Further, pertinent improvement methods were proposed, and a compensation method was proposed for the errors caused by the variation of tip clearance. A new denoising and shaping algorithm based on ensemble empirical mode decomposition (EEMD) was introduced for the errors caused by background noise. Additionally, an optimal installation position of the sensor was also proposed for the errors caused by the asymmetry of the blade tip shape. Finally, simulations and experiments were used to demonstrate the improved methodology. The results show that the measurement error on vibration amplitude and vibration frequency using the proposed method is less than $2.89 \%$ and $0.17 \%$, respectively, which is much lower than the traditional method $(24.44 \%$ and $0.39 \%$, respectively).
\end{abstract}

\section{Introduction}

High-speed rotating blades are the core mechanical components in turbomachinery. Monitoring its vibration state has always been a research hotspot [1-3]. The blade tip timing technique is considered the most promising blade vibration monitoring method because of its noncontact and simplicity $[4,5]$. BTT technology records the arrival times of the blade tip by sensor probes mounted on the equipment casing; then, the blade vibration parameters can be obtained by using these time series [6]. Thus, it is the key of BTT technology to measure these time series accurately. Nowadays, the optical, capacitive, and inductive sensors can be used as BTT probes. An extremely clean probe surface is required for optical sensors [7]. Generally, the capacitive sensor is applied to specific clean working conditions, because it is easily influenced by gas medium [8]. Compared with the two sensors mentioned above, the inductive sensor is more suitable for the industrial environment [9]. Therefore, this paper will mainly study the application of the BTT technique with the eddy current sensor. In the actual measurement, no matter which kind of sensors are used, the original pulse signals are always disturbed by background noise or other factors, which means that BTT signals extracting accurately is a formidable task. This is why the BTT technique has successfully demonstrated its potential, but few high-quality data are still available [10]. Russhard and Back [11, 12] analyzed BTT uncertainties and proposed solutions only for optical probes. Reference [13] proposed a pulsed eddy current signal denoising method, which can remove the noise, but how to accurately extract the pulse rising edge has not resolved. References [14-17] conducted similar research. However, to date, little research has been done on the measurement error of the BTT technique based on eddy current sensors. Thus, how to get a good BTT pulse signal based on eddy current sensors is still a big challenge.

This paper focuses on the fact that it is difficult to extract the BTT signal based on eddy current sensors accurately. The paper is organized as follows. In Section 2, the principle of the BTT technique is briefly introduced. Section 3 analyzes the 
error of the BTT technology using an eddy current sensor. In view of each kind of error, the pertinent improvement methods are proposed in Section 4. In Section 5, the experiments are carried out to demonstrate the effectiveness and the superiority of the proposed improved method. Finally, some major conclusions are drawn in Section 6.

\section{Principle of Blade Tip Timing}

The principle of blade tip timing is shown in Figure 1. Several BTT probes (probes 1, 2, and 3 depicted in the figure) are embedded in a stationary casing around a disk with $\mathrm{K}$ blades. An additional probe $S$ is installed as a reference probe close to the shaft. The time of each blade tip and shaft phase crossing these probes is recorded by means of a series of pulse signals [18]. In Figure 1, channel 1, channel 2, channel 3, and channel $S$ record the pulse data of probe 1, probe 2, probe 3 , and probe $S$, respectively. Channel SV represents the virtual reference pulse according to the pulse data of probe $S$ and the number of blades [19]. The front edge of the pulse signal indicates the arrival time of the blade tip through the probe. Once the blade vibration occurs, the arrival time will change and form a time difference $\Delta t$. Then, the blade vibration magnitude can be calculated by the time difference.

The vibration displacement $l$ can be calculated using (1).

$$
l=V \times \Delta t
$$

where $\Delta t$ represents the time difference caused by blade vibration, and $V$ is the linear velocity of the blade tip.

\section{Error Analysis of BTT Technology with Eddy Current Sensor}

As can be seen from the principle of BTT, the measurement accuracy of the blade vibration response is mainly dependent on the extracting precision of tip arrival times. In order to clarify the response characteristics of the eddy current sensor, calibration tests were carried out. The process of the rotating blade passing the eddy current sensor can be divided into a series of steps, as shown in Figure 2(a). The response amplitudes at different times can be obtained when the blade slowly sweeps across the eddy current probe, and the calibration curve was fitted with the Fourier series using these calibration points as shown in Figure 2(b). Obviously, the fitting curve is continuous and smooth, that is, the actual measured BTT signal is not a standard rectangular pulse and needs further processing.

For the processing of this gradient pulse signal, the traditional method is compared with the given threshold. The time corresponding to the first sampling point larger than the threshold is regarded as the time the blade tip reaches the sensor. However, this simple processing method will bring great errors to the measurement results. The relationship between blade vibration displacement $\Delta s$ (adjacent sampling points), blade rotation diameter $d$, rotation speed $n$, and sample frequency $F s$ is presented in (2).

$$
\Delta s=\frac{\pi \times d \times n}{60 F s} .
$$

Supposing that $n$ is 6000 revolutions per minute, $d$ is $0.5 \mathrm{~m}$, and $F s$ is $100 \mathrm{kHz}$, then the $\Delta s$ will be $0.157 \mathrm{~mm}$. However, the finite element method (FEM) analysis shows that the blade tip vibration displacement at that maximum speed range is $0.13 \mathrm{~mm}$. That means a large error will be generated from the inaccurate extraction of the BTT pulse signal. Thus, the accurate extraction of tip timing pulse signals is the key to ensure the measurement accuracy of the BTT system.

By analyzing the BTT technique with an eddy current sensor, it can be clearly seen that the main factors affecting the BTT measurement accuracy can be summarized as follows: the variation of the blade tip clearance, the interference of background noise, and the asymmetry of the blade tip shape.

3.1. Error Caused by the Variation of Blade Tip Clearance. The variation of tip clearance is unavoidable due to such things as manufacture, installation, and vibration. In traditional BTT pulse signal extraction, a threshold value is set as a criterion. Once the amplitude of the rising edge of the pulse exceeds the threshold value, the arrival time of blade tip crossing probes can be determined. However, with a change of tip clearance, the feedback signal will also be changed, as shown in Figure 3. The rising edges of the pulse signal will shift forward or backward, and the measuring error caused by the tip clearance will be introduced into the BTT system. In Figure $3(\mathrm{a}), d_{1}, d_{\mathrm{c}}$, and $d_{3}$ represent various clearance values between the blade tip and probe, and $d_{c}$ denotes the calibration value. In Figure 3(b), C1 and C3 represent the corresponding impulse response curves at different clearances of $d_{1}$ and $d_{3}$, respectively. $\Delta h_{1}$ and $\Delta h_{3}$ denote the amplitude difference between the clearances of $d_{1}$ and $d_{3}$ relative to the calibration clearance $d_{\mathrm{c}}$. Correspondingly, $\Delta$ $t_{1}$ and $\Delta t_{2}$ denote the time difference owing to the difference in the blade tip clearance. The vibration measurement error $l_{c}$ can be described in (3).

$$
l_{c}=V \bullet \Delta t_{1} \text { or } l_{c}=V \bullet \Delta t_{2}
$$

3.2. Error Caused by Background Noise Interference. Among the error factors of the BTT technique, the largest is background noise, due to its characteristics of randomness and uncertainty. We know that all measurement systems can potentially be affected by noises, but compared with the traditional test system, the BTT system is more sensitive. After analyzing and testing in the test rig, three aspects of the BTT noise sources could be identified: (a) periodic or aperiodic shocking signal produced by the casing vibration, (b) random noise produced by impurity in the gas, and (c) periodic clutter waves produced by the collector system.

The test experiments were conducted on the fan rig as shown in Figure 4, and the parameters of the test rig are shown in Table 1. In order to simulate real operating conditions, impure gas was blown onto the blade. The original pulse signal was measured by eddy current probes (Bently, 330903). The output of the sensors is collected by the DAQ (DT9857E). 


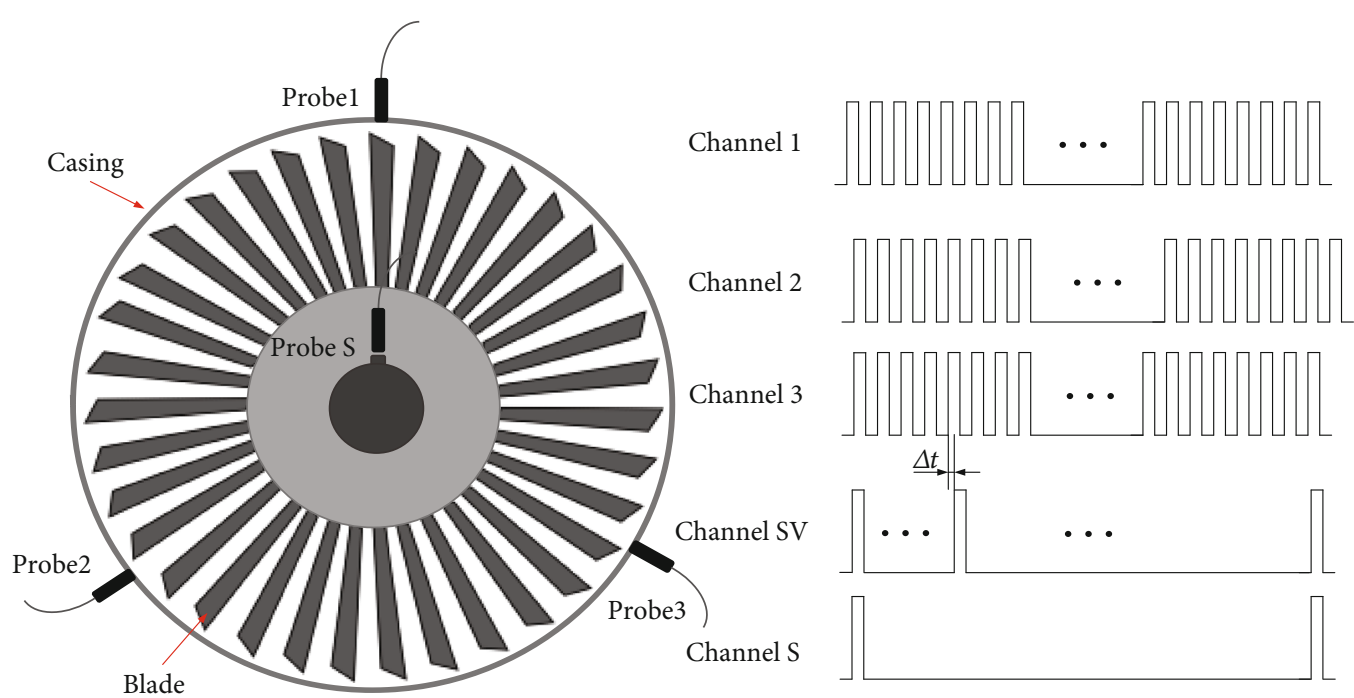

FIgURE 1: Measurement principle of blade tip timing systems.

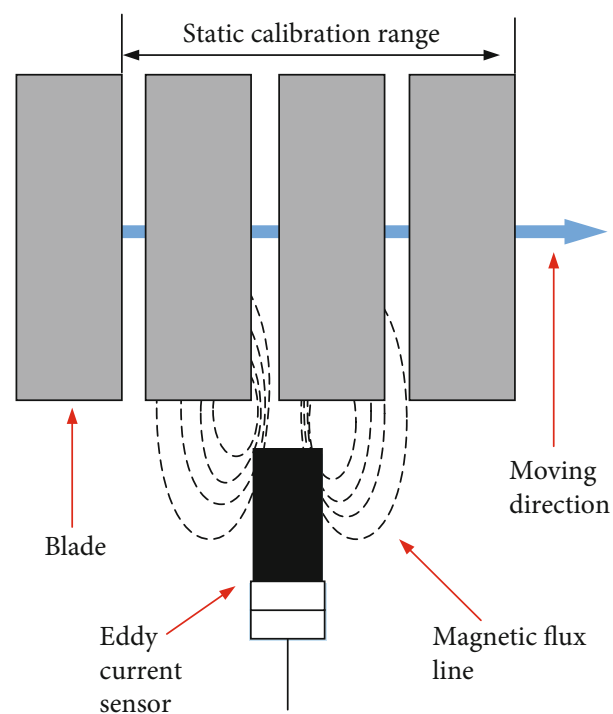

(a)

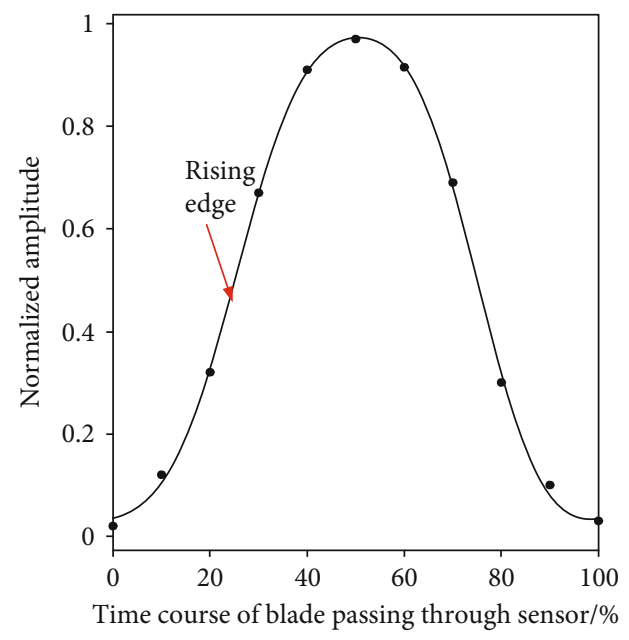

(b)

FIGURE 2: Calibration for BTT sampling process.

The measured BTT signals (normalized) are shown in Figure 5. It can be seen that the pulse amplitude varied greatly, indicating that the tip clearance was different, and there were periodic clutter and random noise interference. Therefore, it was difficult to accurately extract the arrival time of the blade tip crossing probes under the interference of noise. Furthermore, it was difficult to accurately time the blade tip by using the pulse rising edge under noise interference. Thus, the research on using the BTT signal extracting method with background noise is meaningful.

3.3. Error Caused by Asymmetry of Blade Tip Shape. Another measurement error is caused by the asymmetry of the blade tip shape. The diverse response signals can be recorded when the different tip shape sweeps through the eddy current sensor. When the tip shape is symmetrical, a symmetrical response signal will be obtained as shown in Figure 6(a).
When the tip shape is asymmetrical, an asymmetrical response signal will be obtained, as shown in Figure 6(b). Thus, the measurement error $l_{s}$ will be introduced due to the asymmetrical tip shapes, which can be described in (4):

$$
l_{s}=V \bullet \Delta t_{3} .
$$

\section{Improvement Methods}

4.1. Improvement for the Variation of Blade Tip Clearance. The working principle of eddy current sensors is based on the electromagnetic induction phenomenon. The highfrequency oscillating current is transmitted by the front-end device to the eddy current probe, which generates a high frequency alternating magnetic field at the front of the probe. When the measured conductor enters this alternating magnetic field, a reverse alternating magnetic field will be 


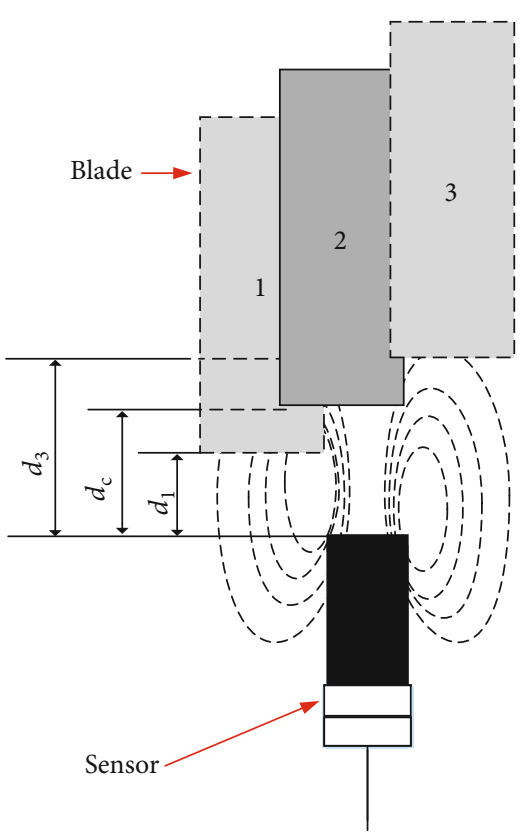

(a)

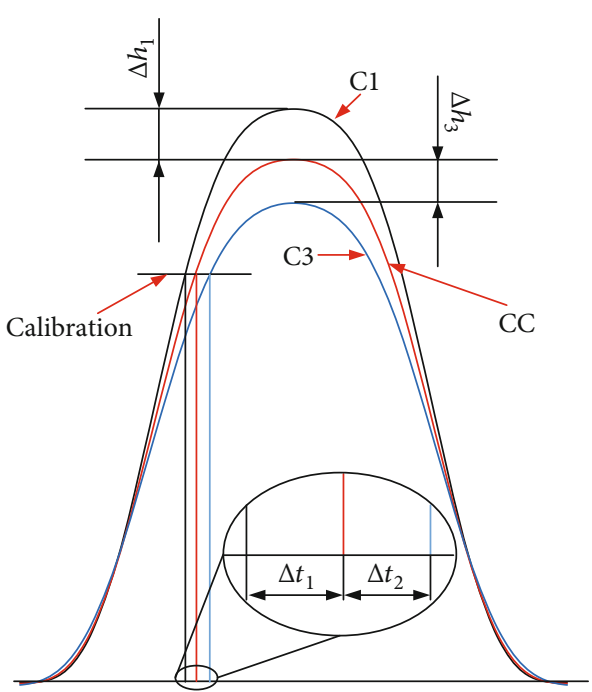

(b)

FIGURE 3: Measuring error introduced by tip clearance variation.

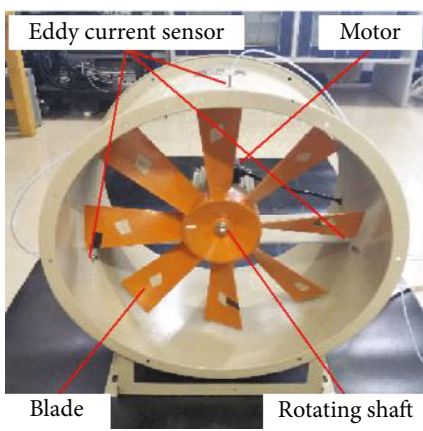

(a) Major structure

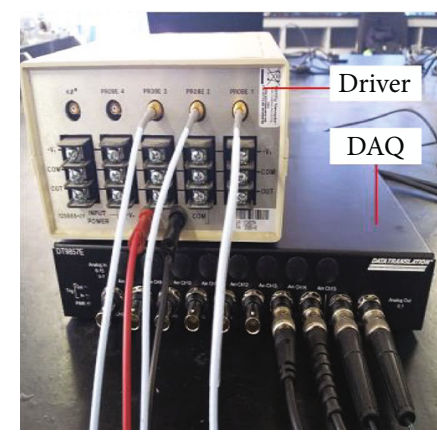

(b) Auxiliary structure

FIgURE 4: Test rig.

TABLE 1: Test rig parameters.

\begin{tabular}{lc}
\hline Parameters & Properties \\
\hline Material & Al7075 \\
Blade length & $175 \mathrm{~mm}$ \\
Width (tip/root) & $84 \mathrm{~mm} / 58 \mathrm{~mm}$ \\
Poisson's ratio & 0.33 \\
Blade thickness & $2.5 \mathrm{~mm}$ \\
\hline
\end{tabular}

generated on the surface of the conductor, which will change the amplitude and phase of the high-frequency oscillating current in the original sensor. This change has a good linear relationship in the measurement range. Based on this, the distance between the measured conductor and the sensor can be accurately measured. Similarly, the variation of tip clearance will be linearly related to the impulse response height. Then, the error caused by the variation of blade tip clearance can be eliminated by compensating tip timing information with tip clearance information.

In order to explore the relationship between tip timing and tip clearance, experiments were carried out on the test rig, as shown in Figure 4. The spiral micrometer was used to adjust the clearance value, and the adjustment range of the tip clearance value was set to $0.5 \sim 2.5 \mathrm{~mm}$. At the same time, in order to ensure that the tip clearance measurement system was not damaged, the installed sensor needed to be connected to the casing liner. The measurement accuracy is required to reach the micron level. Nine groups of experiments were carried out using $l_{1.5}$ (tip timing value with $1.5 \mathrm{~mm}$ tip clearance) as a benchmark. To ensure the measurement precision, each group of experiments was repeated 10 times, and the mean values of 10 experiments were taken as the error values under a certain gap. The experimental results are shown in Figure 7. Here, the calculation equation 


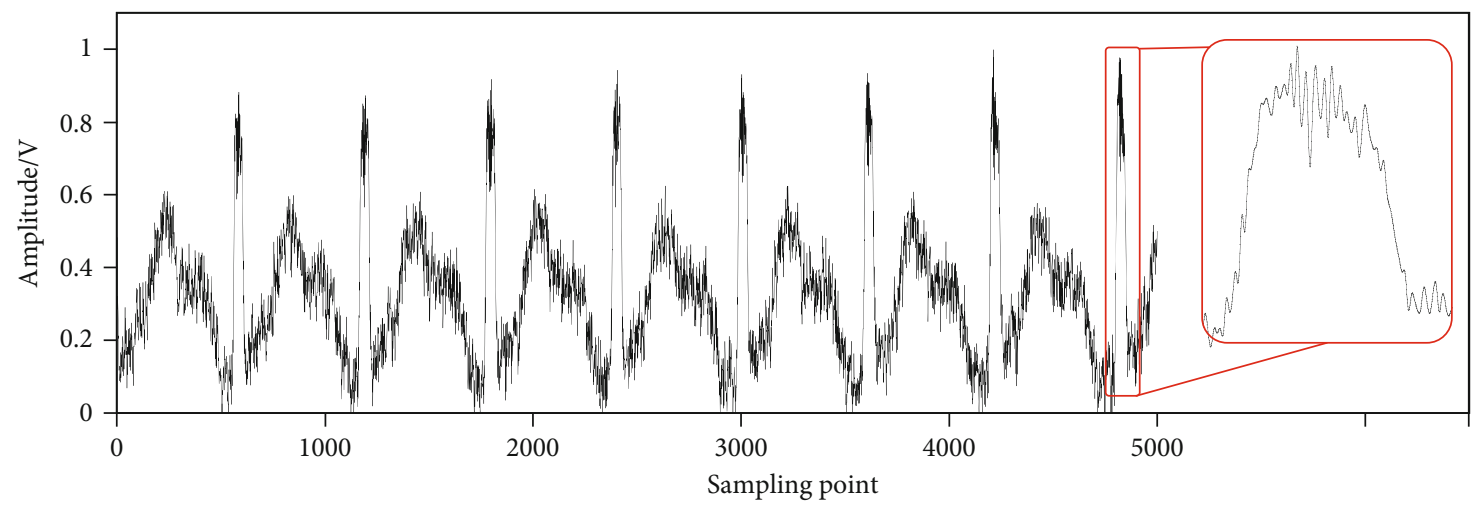

Figure 5: Blade tip timing signal.

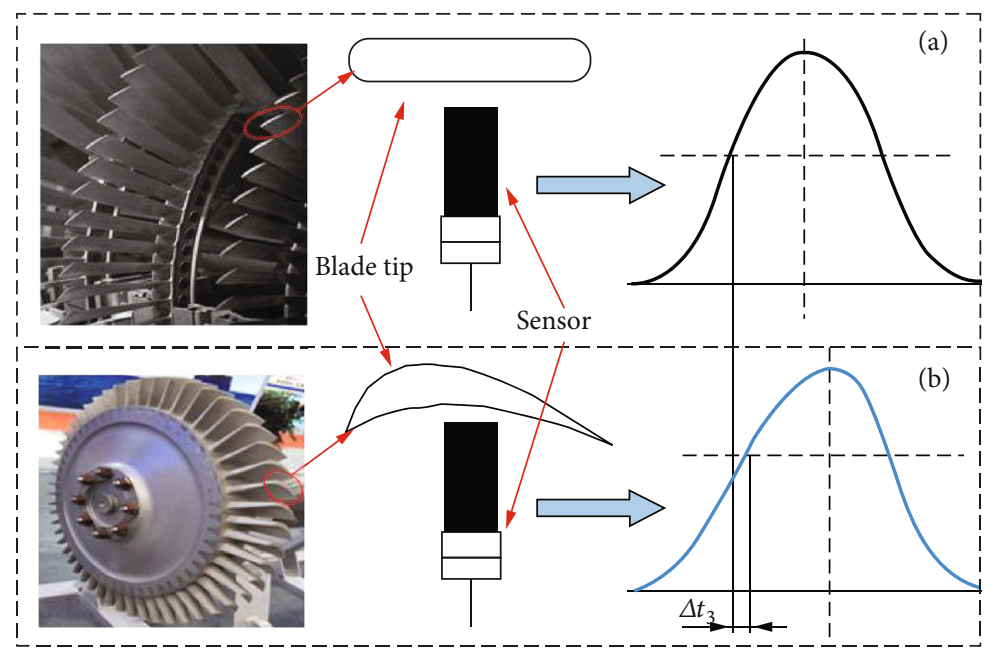

FIGURE 6: Measuring error introduced by the different blade tip shapes.

of the relative error ratio (RER) is as follows.

$$
\mathrm{RER}_{i}=\frac{l_{i}-l_{1.5}}{l_{1.5}} \times 100 \%,
$$

where $l_{i}$ is the tip timing value with $i \mathrm{~mm}$ tip clearance $(i=0.5,0.75,1, \ldots, 2.5)$.

As can be seen from Figure 7, within the linear measurement range of the eddy current sensor, the error decreases linearly with the increase of tip clearance. This shows that, as long as the relationship between the tip clearance and measurement error is obtained by calibrating the measured blades, the measurement error can be compensated to eliminate the measurement error and obtain the accurate vibration amplitude of the blades. The experimental fan is tested, and the blade vibration under different tip clearance is measured by using $1.5 \mathrm{~mm}$ clearance as a reference value. The comparison between the traditional method and the error compensation method is obtained as shown in Figure 8. Obviously, the measurement error caused by the variation of tip clearance can be effectively eliminated by the introduced error compensation method.

\subsection{Improvement for Background Noise Based on EEMD and Square-Wave Shaping}

4.2.1. Ensemble Empirical Mode Decomposition (EEMD). EEMD is a time-domain signal analysis method widely used in nonlinear and nonstationary signal processing [20-22]. Unlike the Fourier transform and wavelet transform, EEMD can decompose the original signal adaptively according to its characteristics. The noisy signal $x(t)$ will be adaptively decomposed into $m$ intrinsic modes $\operatorname{IMF}(t)$ and a residue $r_{m}(t)$ from high to low frequency [23]; the decomposition process is described as follows:

$$
x(t)=\sum_{k=1}^{m} \operatorname{IMF}_{k}(t)+r_{m}(t) .
$$

Based on the above decomposition, the multiscale filters, high-pass (HP), band-pass (BP), and low-pass (LP), can be designed, which can be expressed as (7)-(9).

$$
\mathrm{HP}_{p}=\sum_{k=1}^{p} \operatorname{IMF}_{k}(t)
$$




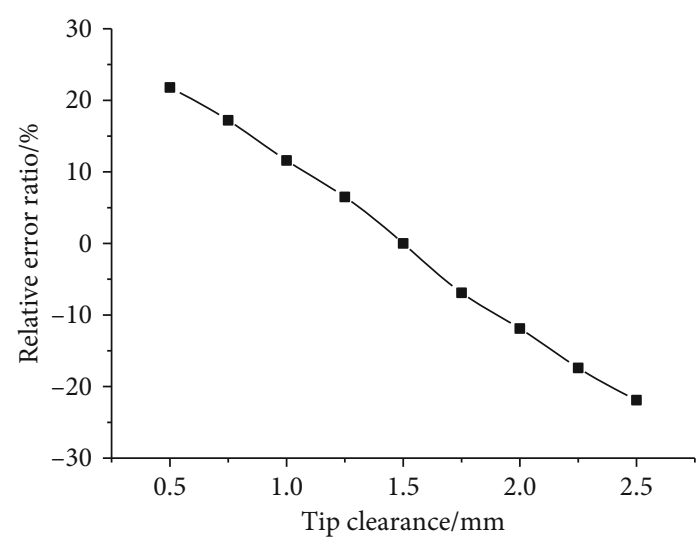

Figure 7: Relative error ratio under different tip clearance.

$$
\begin{gathered}
\mathrm{BP}_{b}^{q}=\sum_{k=b}^{q} \operatorname{IMF}_{k}(t), \\
\mathrm{LP}_{l}=\sum_{k=l}^{m} \operatorname{IMF}_{k}(t)+r_{m}(t),
\end{gathered}
$$

wherep, $q, b$, and $l$ are the selection parameters of intrinsic modes for different scale filters, and these values are determined by noise reduction requirements. For the BTT pulse signal, the effective information is concentrated in low-frequency components.

4.2.2. Square-Wave Shaping. After noise reduction, the nonstandard pulse signal needs to be converted into the standard. Therefore, a square-wave shaping algorithm is proposed, which mainly includes three processing steps: (a) signal normalization (SN), (b) square-wave shaping (SWS), (c) exceptional signal removal.

The detailed procedure of the algorithm is as follows: firstly, the original signal will be normalized in the SN step. Then, according to the magnitude of the signal, it can be divided into three parts: $[0,0.4),[0.4,0.6)$, and $[0.6,1]$. The signal in $[0,0.4)$ is set to $0,[0.6,1]$ is set to 1 , and for signal in $[0.4,0.6)$, three adjacent sampling points are selected; if the mean value is greater than 0.45 , set to 1 , otherwise set to 0 . The above parameters were obtained by repeated experiments. It should be noted that after the SWS processing, the narrowband pulse signal needs to be removed.

In order to evaluate the performance of the SWS method, the index Mean Square Error (MSE) and the correlation coefficient $\rho$ are introduced. They represent the approximation and correlation of signals before and after processing, respectively. The corresponding formulas are shown in (10)-(11).

$$
\mathrm{MSE}=\sqrt{\frac{\sum_{j=1}^{z}\left(\widehat{x}_{j}-x_{j}\right)^{2}}{z}},
$$

$$
\rho=\frac{\operatorname{cov}\left(\widehat{x}_{j}, x_{j}\right)}{\sqrt{D\left(\widehat{x}_{j}\right)} \sqrt{D\left(x_{j}\right)}},
$$

where $z$ denotes the signal length, $x_{j}$ is the raw signal, and $\widehat{x}_{j}$ indicates the processed signal. $\operatorname{cov}\left(\widehat{x}_{j}, x_{j}\right)$ denotes the covariance of $\widehat{x}_{j}$ and $x_{j}, D\left(\widehat{x}_{j}\right)$ and $D\left(x_{j}\right)$ are the standard deviation. Considering that MSE may be equal to 0 , we define $(\mathrm{MSE}+1)^{-1}$ as the judge index of the processing effect, which can be expressed as $\mathrm{MSE}^{-1}$. Obviously, $0<\mathrm{MSE}^{-1}<1$, and the smaller MSE is, the closer $\mathrm{MSE}^{-1}$ is to 1 , and a better processing effect is.

Because the similarity and correlation need to be considered simultaneously in this step, therefore, an objective function is defined as $\max \{f\}$, and the equation is as follows.

$$
\max \{f\}=\max \left\{\alpha \operatorname{MSE}^{-1}+(1-\alpha)|\rho|\right\},
$$

where $\alpha$ and 1- $\alpha$ denote the important factors of $\mathrm{MSE}^{-1}$ and $\rho$ , respectively. Because they are equally important in error description, the important factor $\alpha$ is set to 0.5 .

4.2.3. Application. By analyzing the rotating blade and BTT system, the measured BTT signal components can be divided into three categories: standard pulse signal, periodic clutter signal, and Gaussian white-noise signal [24] Then, the noisy BTT signal $x(t)$ can be expressed as follows.

$$
x(t)=s(t)+c(t)+n(t)
$$

where $s(t)$ indicates the rectangular pulse signal, $c(t)$ denotes the periodic waveform, and $n(t)$ represents the white Gaussian noise signal.

To verify the universality and feasibility of the proposed method, the amplitude of the periodic waveform $c(t)$ is set to 0.15 , and the energy of Gauss white noise is set to 0.5 . The subsignals and synthetic signals are shown in Figure 9. Obviously, the original rectangular pulse signal has been submerged by noise.

The noisy pulse signal $x(t)$ is adaptively decomposed into 10 intrinsic modes, $\mathrm{IMF}_{1}-\mathrm{IMF}_{10}$, and residue $r_{11}$ according to Eq. (5), and the decomposition results are shown in Figure 10. Then, a series of multiscale filters are established according to Eq. (8), and the filtering effect is shown in Figure 11.

Then, square wave shaping is performed on the above denoised signal, and the shaping result is shown in Figure 12. The black signal represents the rectangular pulse signal $s(t)$, and the blue signal is the shaping signal. Comparing the shaping signal with the initial noise-free pulse signal, the shaping result by $\mathrm{LP}_{4}$ yields the best one, followed by $\mathrm{LP}_{5}$ and $\mathrm{LP}_{3}$.

The index $\mathrm{MSE}^{-1}, \rho$, and $\max \{f\}$ under different scale shaping effects are analyzed, and the result is shown in Figure 13. The largest value of $\mathrm{MSE}^{-1}, \rho$, and $\max \{f\}$ are all in $\mathrm{LP}_{4}$ and are 0.9782, 0.9294, and 0.8805, respectively. Therefore, $\mathrm{LP}_{4}$ is the optimal noise reduction filter. 


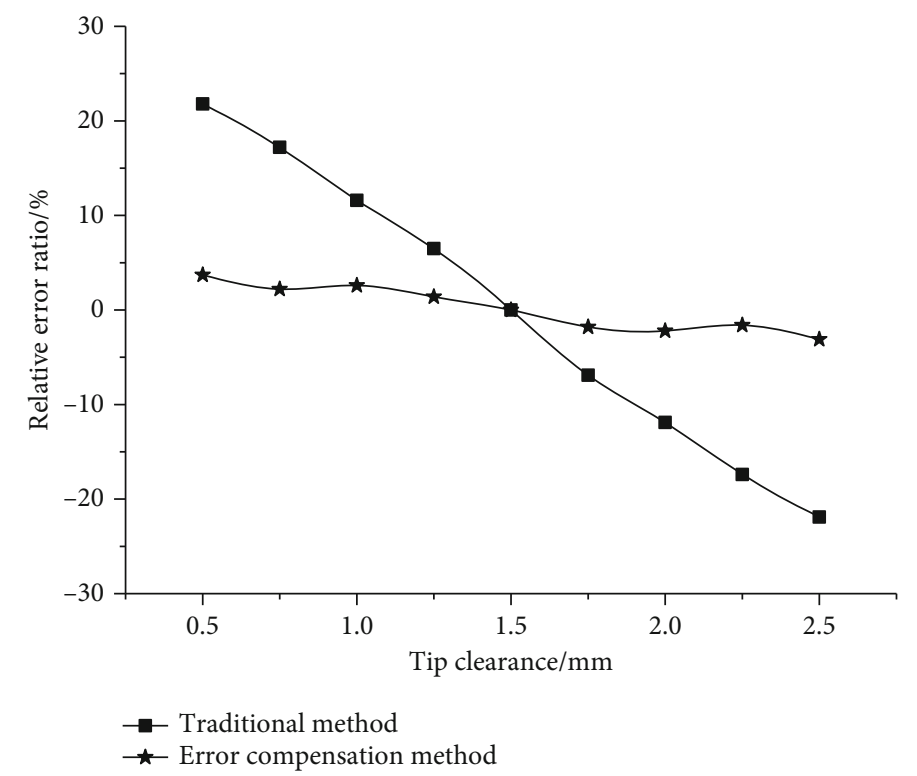

FIgURE 8: Comparison of the traditional and error compensation method.

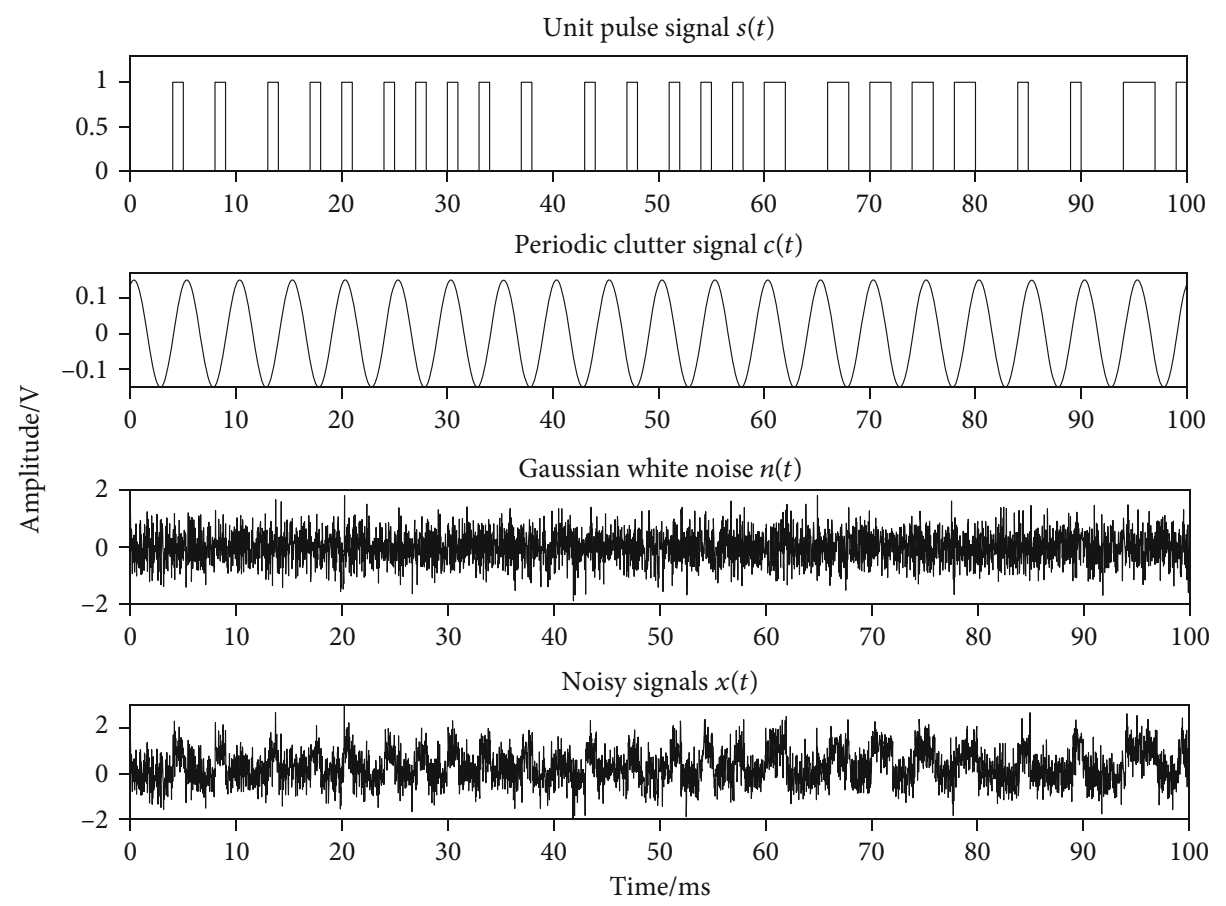

Figure 9: Noisy BTT impulse signal.

The optimal denoising and shaping algorithm $\mathrm{LP}_{4}$ is used to process the measured BTT signal, and the result is shown in Figure 14. Obviously, the noise effect is effectively eliminated.

4.3. Improvement for the Asymmetry of Blade Tip Shape. To investigate the error caused by the asymmetry of blade tip shape, many experiments on different installation positions of sensors have been carried out as shown in Figure 15(a). The results show that, due to the asymmetry of the tip, it is difficult to maintain the symmetry of the impulse response measured by the eddy current sensor. However, the measurement error is smallest at the tangent parallel position of the blade tip edge, as shown in Figure 15(b).

\section{Validation}

Ten groups of experiments (the test rig is shown in Figure 4) and a finite element simulation (ADAMS software) were carried out to verify the feasibility of the improved method. 

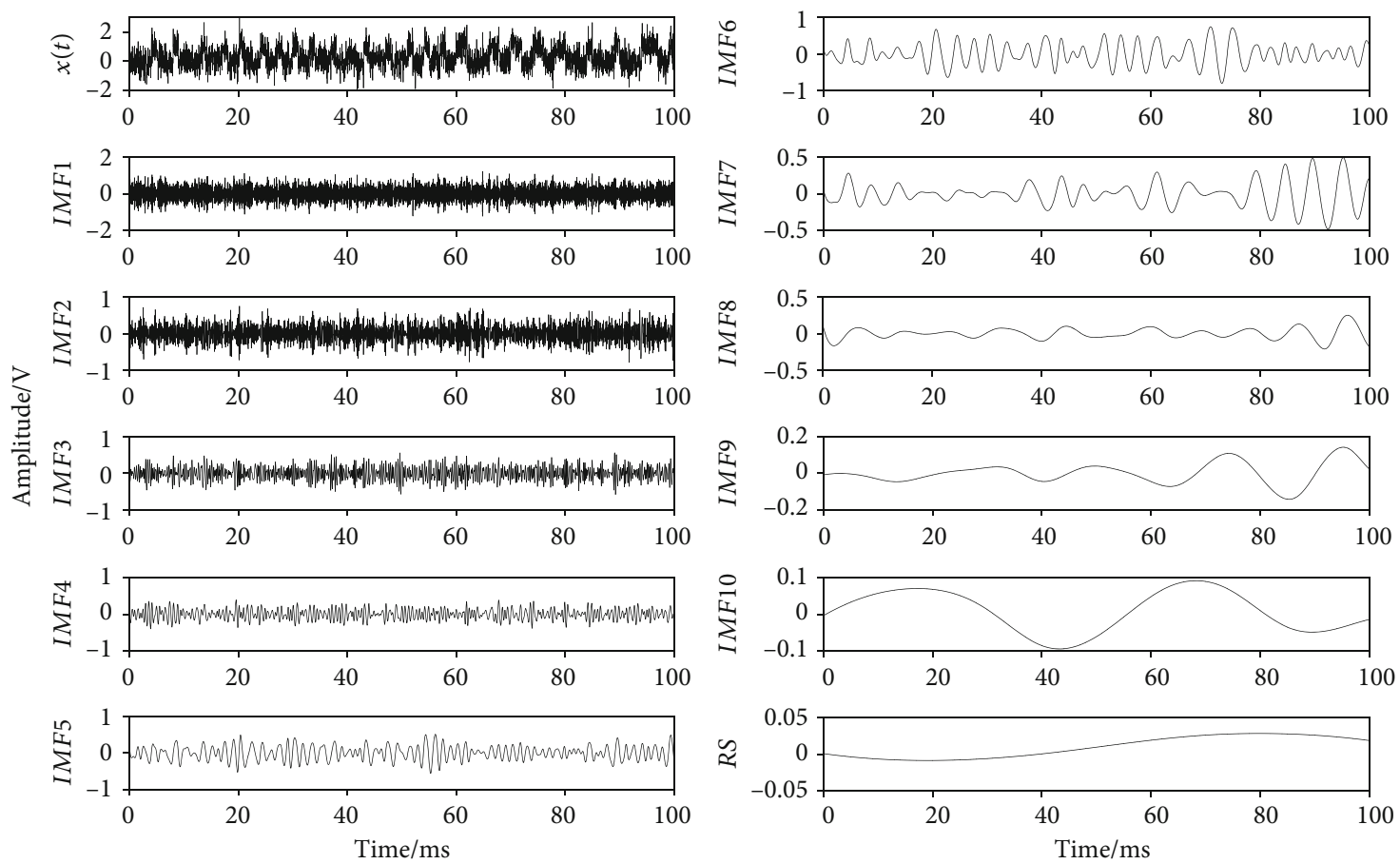

FIgURE 10: Noise signal decomposition.
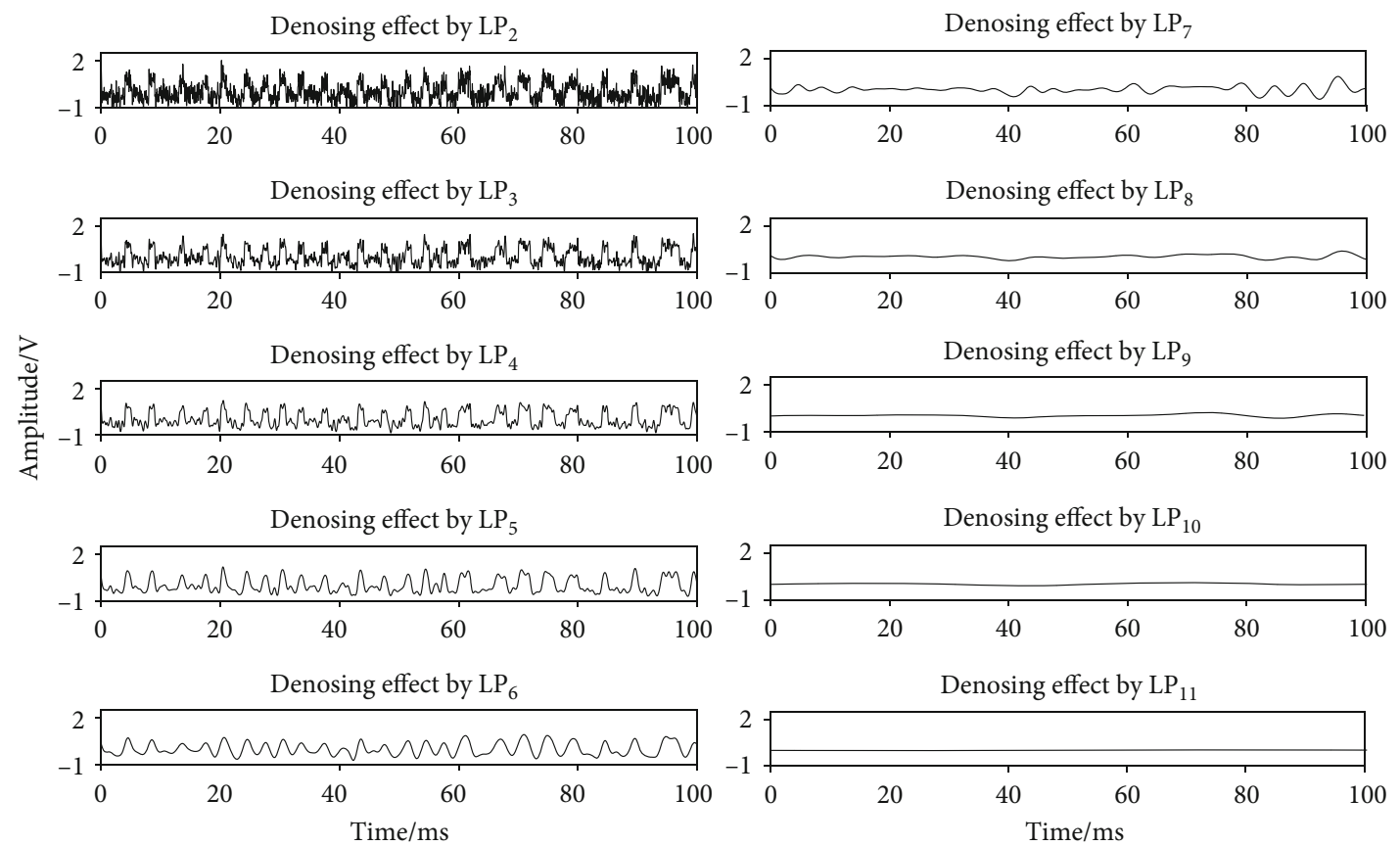

FIgURE 11: Noise reduction effect of different scale filters.

The experimental settings are shown in Table 2, including the blade cracks at different positions and of different sizes. Other parameters of the test rig were set as shown in Table 1 (Section 3.2). The experimental procedure is as follows. Firstly, the original pulse signals in various states were collected, then processed by the improved method. Secondly, the response signals under various states were obtained by finite element simulation. Finally, the experimental and simulation signals were compared, and the main parameters include the vibration frequency of the blade vibration amplitude. The comparative results are shown in Figure 16.

As can be observed in Figure 16, in terms of the vibration amplitude, the traditional BTT method has a large measurement error, the maximum being $24.44 \%$ and the average 
Shaping effect by $\mathrm{LP}_{2}$

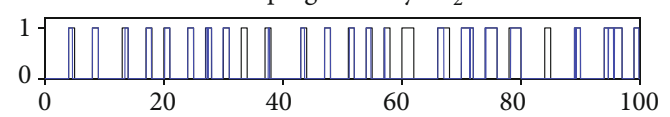

Shaping effect by $\mathrm{LP}_{3}$
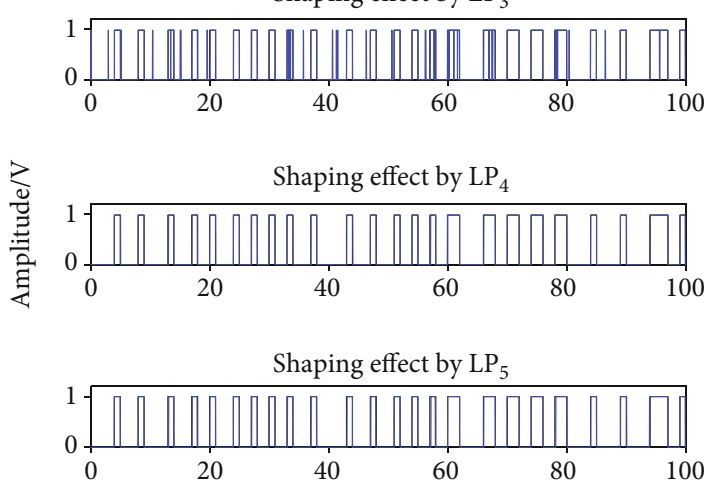

Shaping effect by $\mathrm{LP}_{6}$

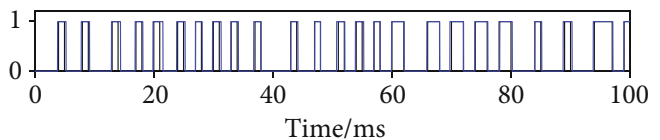

Time/ms

_ Noise-free signal

- Shaping effect
Shaping effect by $\mathrm{LP}_{7}$

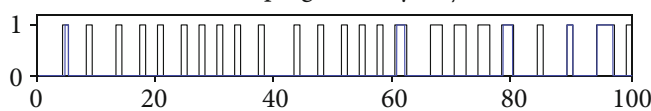

Shaping effect by $\mathrm{LP}_{8}$

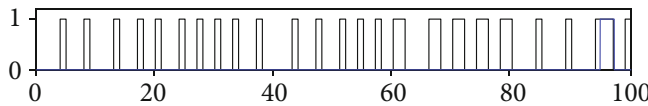

Shaping effect by $\mathrm{LP}_{9}$

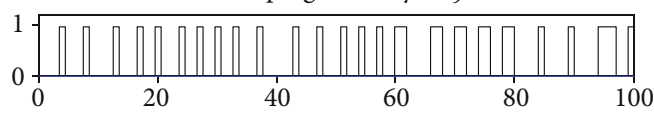

Shaping effect by $\mathrm{LP}_{10}$

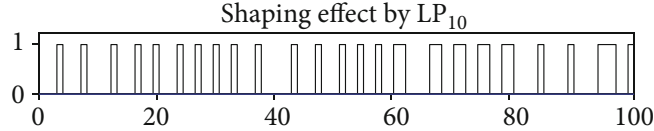

Shaping effect by $\mathrm{LP}_{11}$

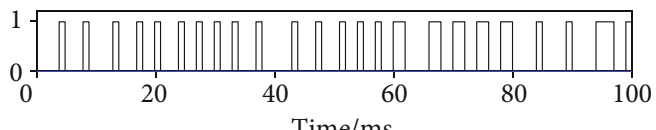

FIGURE 12: Shaping effect of different low-pass filters.

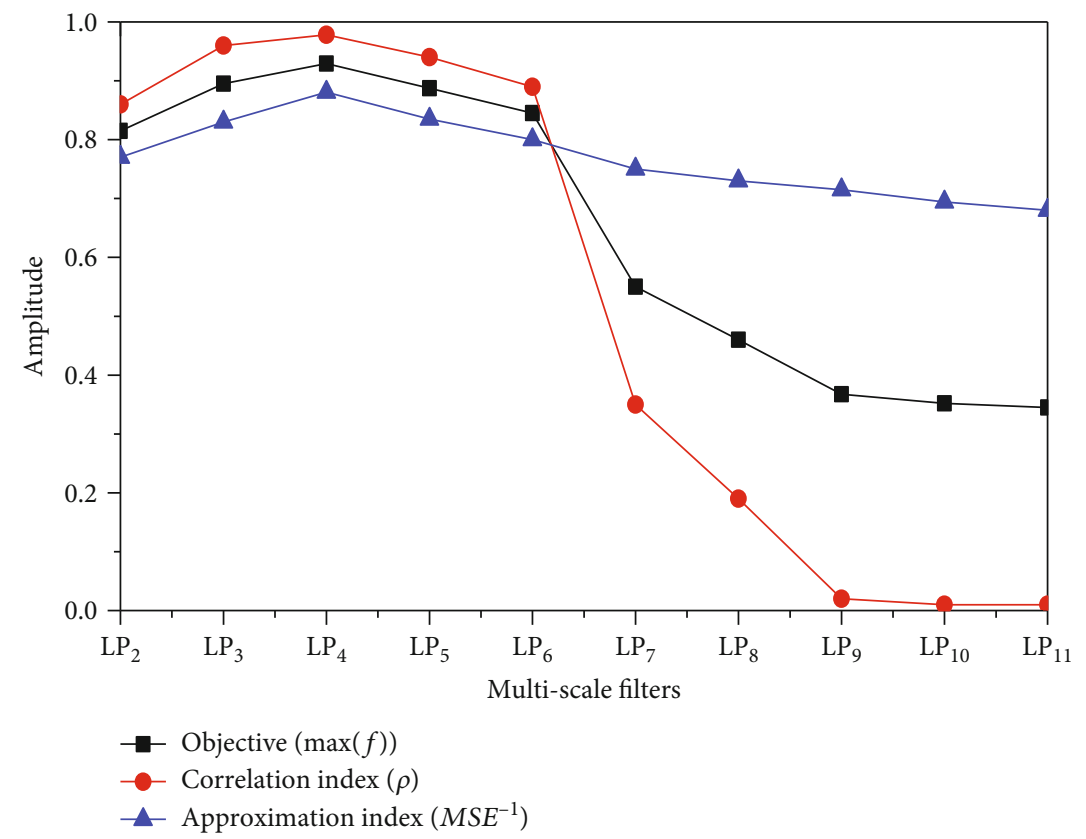

FIGURE 13: Variation trends of the index $\operatorname{MSE}^{-1}, \rho$, and $\max \{f\}$.

error being $18.98 \%$. The improved method clearly reduces the error-the maximum error is only $2.89 \%$ and the average error is only $1.59 \%$-and the measurement accuracy is improved by $22.85 \%$. Thus, the measurement accuracy of vibration amplitude has been greatly improved. In terms of vibration frequency, the maximum measurement error of the traditional BTT method is $0.39 \%$ and the average error is $0.3 \%$, whereas the maximum measurement error of the improved method is $0.17 \%$ and the average error is $0.1 \%$. Compared with the vibration amplitude, the measurement 


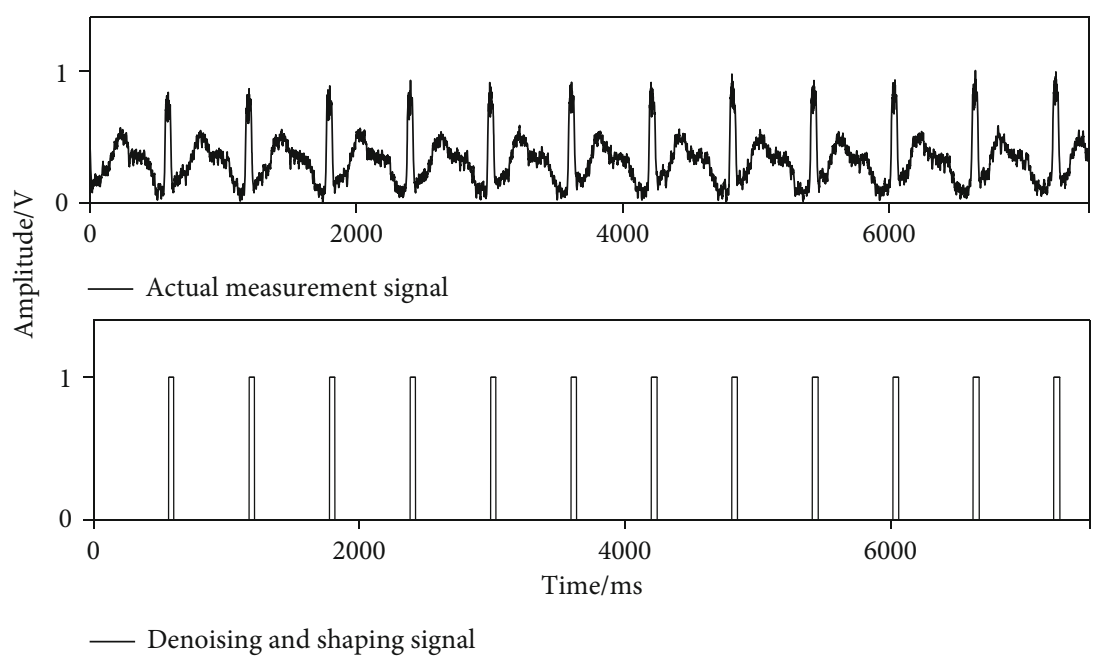

FIGURE 14: Denoising and shaping effect for actual BTT signal.

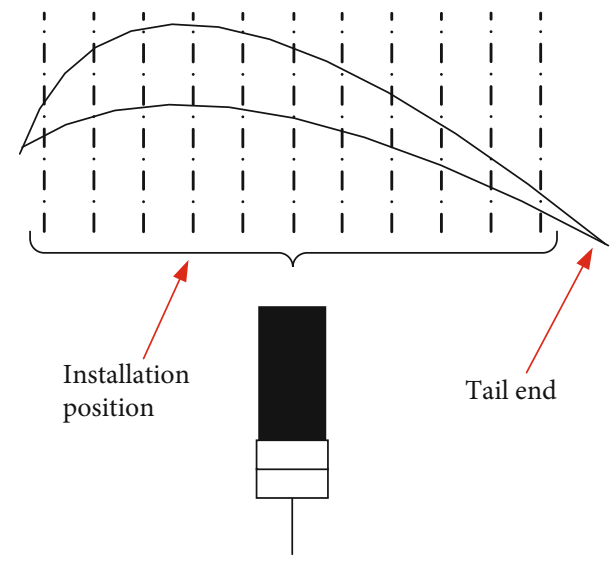

(a)

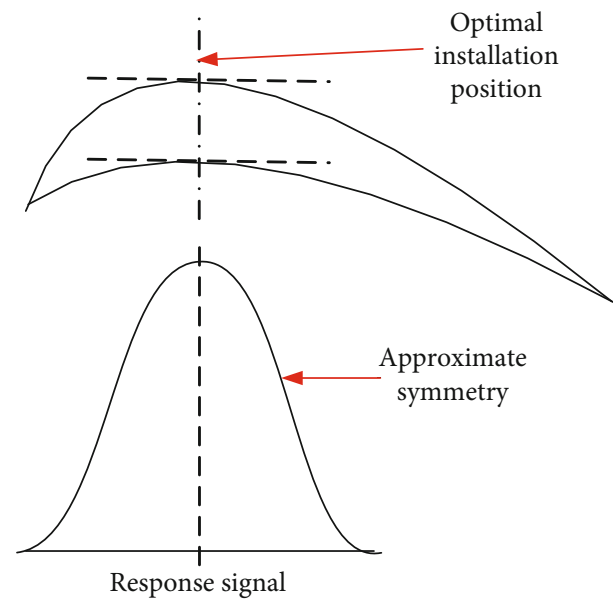

(b)

FIgURE 15: Experiments on the optimal installation position of sensors.

TABLE 2: Experimental settings.

\begin{tabular}{lcccc}
\hline No. & Types & Crack positions & Size $(\mathrm{mm})$ & Fault photo \\
\hline 1 & $\mathrm{~N}$ & None & 0.0 & None \\
2 & $\mathrm{~T} 1$ & & 4.18 & \\
3 & $\mathrm{~T} 2$ & Top & 8.39 & \\
4 & $\mathrm{~T} 3$ & & 12.58 & \\
5 & M1 & & 3.51 & \\
6 & M2 & Middle & 7.06 & \\
7 & M3 & & 10.62 & \\
8 & R1 & & 2.86 & \\
9 & R2 & Root & 5.82 & \\
10 & R3 & & 8.73 & \\
\hline
\end{tabular}

error of vibration frequency is not so high, but the result caused by the error of vibration frequency is more serious. According to the requirement of damage tolerance, the natural frequency of the rotating blade decreases by $0.5 \%$, which means that serious damage occurs. Thus, this measurement error can easily lead to a wrong judgment in relation to the blade state. Therefore, it is very important to improve the measurement accuracy of vibration frequency by $0.2 \%$. In summary, the improved method greatly improves the measurement accuracy of the BTT technology based on the eddy current sensor.

\section{Conclusions}

The causes of measurement error in the BTT system using the eddy current sensor include the variation of blade tip clearance, the interference of background noise, and the asymmetry of the blade tip shape. These factors lead to the advance or delay of the blade arrival time recorded by the 


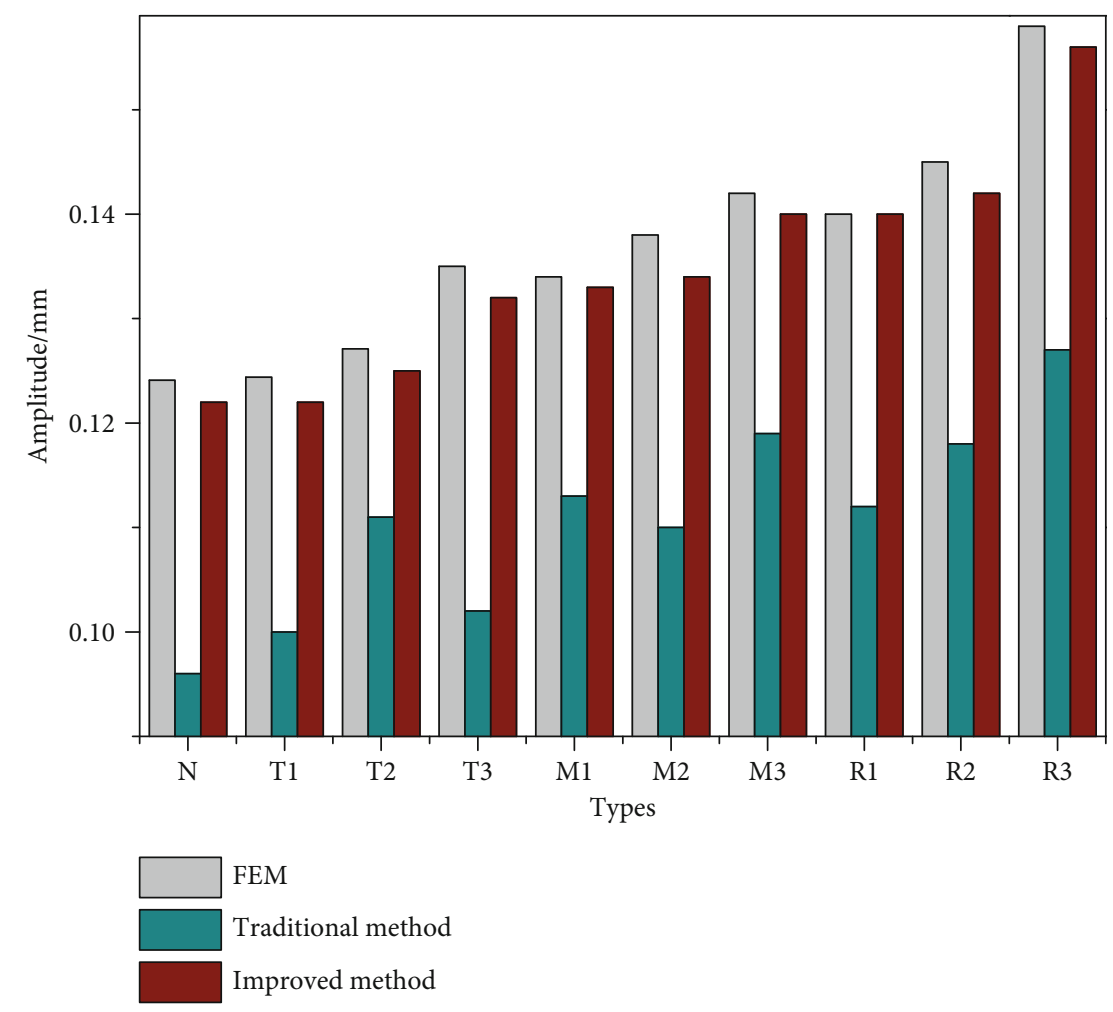

(a) Comparison of vibration amplitude

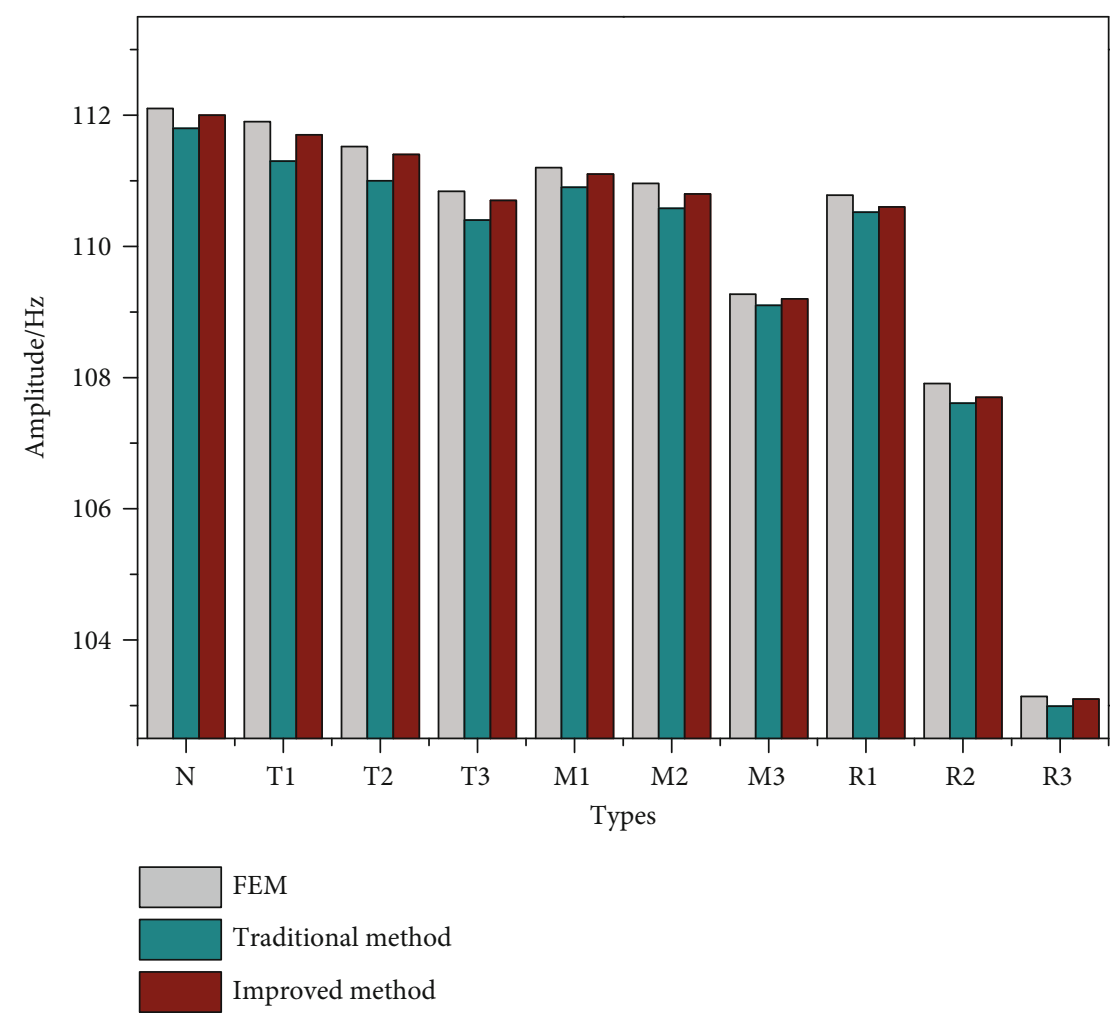

(b) Comparison of vibration frequency

Figure 16: Comparison of vibration response with FEM, traditional method, and improved method. 
BTT system. Aiming at finding the causes of each kind of error, the corresponding solutions are put forward and verified by experiments. The major findings of and conclusions drawn from this study may be summarized as follows.

(1) The relationship between blade vibration displacement (adjacent sampling points), tip rotating diameter, rotating speed, and sampling frequency was deduced, and the influence of measurement error was analyzed

(2) The influence of tip clearance variation on measurement accuracy was analyzed, and an error compensation method was proposed. By coupling tip clearance information to the BTT signal, the measurement error caused by the tip clearance can be eliminated

(3) The influence of noise interference on BTT measurement accuracy was analyzed, and a new algorithm based on EEMD and square-wave shaping was proposed to extract the tip timing signals. A mathematical model was constructed to verify the effectiveness of the algorithm

(4) The influence of the asymmetry of blade tip on BTT measurement accuracy was analyzed, and the optimal installation position of sensors was put forward through experimental research

(5) Finally, a series of experimental tests was carried out to compare the traditional tip timing signal extraction method with the improved method. The experimental results show that the improved method has higher measurement accuracy than the traditional method

Future work will focus on how to extract the tip timing signals accurately under variable operating conditions.

\section{Data Availability}

The data supporting the results of this study can be obtained from the corresponding authors as required.

\section{Conflicts of Interest}

The authors declare that they have no conflicts of interest.

\section{Acknowledgments}

This research was supported by the Science and Technology Plan Projects of State Administration for Market Regulation (2019MK131) and CSEI (2019-Youth-10).

\section{References}

[1] S. Wu, Z. Zhao, Z. Yang, S. Tian, L. Yang, and X. Chen, "Physical constraints fused equiangular tight frame method for blade tip timing sensor arrangement," Measurement, vol. 145, pp. 841-851, 2019.

[2] R. Wu, D. Zhang, Q. Yu, Y. Jiang, and D. E. Arola, "Health monitoring of wind turbine blades in operation using three- dimensional digital image correlation," Mechanical Systems and Signal Processing, vol. 130, pp. 470-483, 2019.

[3] Y. Fu, J. Yuan, S. Yuan, G. Pace, and L. d'Agostino, "Effect of tip clearance on the internal flow and hydraulic performance of a three-bladed inducer," International Journal of Rotating Machinery, vol. 2017, Article ID 2329591, 10 pages, 2017.

[4] R. G. Du Toit, D. H. Diamond, and P. S. Heyns, "A stochastic hybrid blade tip timing approach for the identification and classification of turbomachine blade damage," Mechanical Systems and Signal Processing, vol. 121, pp. 389-411, 2019.

[5] G. Rigosi, G. Battiato, and T. M. Berruti, "Synchronous vibration parameters identification by tip timing measurements," Mechanics Research Communications, vol. 79, pp. 7-14, 2017.

[6] H. Guo, F. Duan, and J. Zhang, "Blade resonance parameter identification based on tip-timing method without the onceper revolution sensor," Mechanical Systems and Signal Processing, vol. 66-67, pp. 625-639, 2016.

[7] M. Neumann, F. Dreier, P. Günther et al., "A laser-optical sensor system for blade vibration detection of high-speed compressors," Mechanical Systems and Signal Processing, vol. 6465, pp. 337-346, 2015.

[8] C. F. Huang and M. J. Hou, "Technology for measurement of blade tip clearance in an aeroengine," Measurement and Control Technology, vol. 27, pp. 27-32, 2011.

[9] K. S. Chana, M. T. Cardwell, and J. S. Sullivan, "The development of a hot section eddy current sensor for turbine tip clearance measurement,", in Proceeding of ASMS Turbo Expo, pp. 35-46, Texas, USA, 2013.

[10] P. Russhard, "Blade tip timing (BTT) uncertainties," AIP Conference Proceeding, vol. 1740, article 020003, 2016.

[11] P. Russhard, "BTT data zeroing techniques," Proceedings of the 59th International Instrumentation Symposium and 2013 MFPT Conference, , pp. 1-46, The International Society of Automation, Cleveland, OH, USA, 2013.

[12] P. Russhard and J. D. Back, "Rotating blade analysis: U.S. Patent," 2015, Patent 9,016,132.

[13] C. Huang, W. Xinjun, X. Zhiyuan, and Y. Kang, "Pulsed eddy current signal processing method for signal denoising in ferromagnetic plate testing," NDT \& E International, vol. 43, no. 7, pp. 648-653, 2010.

[14] N. Jamia, M. I. Friswell, S. el-Borgi, and P. Rajendran, "Modelling and experimental validation of active and passive eddy current sensors for blade tip timing," Sensors and Actuators A: Physical, vol. 285, pp. 98-110, 2019.

[15] Z. Kubín, T. Mísek, J. Hlous, T. Dadaková, J. Kellner, and T. Bachorec, "Calibration of blade tip-timing sensor for shrouded 40" last stage blade," Mechanical Systems and Signal Processing, vol. 108, pp. 88-98, 2018.

[16] S. Madhavan, R. Jain, C. Sujatha, and A. S. Sekhar, "Vibration based damage detection of rotor blades in a gas turbine engine," Engineering Failure Analysis, vol. 46, pp. 26-39, 2014.

[17] H. W. Li, F. Han, Y. W. Ma, H. C. Wang, Z. Y. Zhou, and Z. Tao, "Experimental investigation on the effects of rotation and the blowing ratio on the leading-edge film cooling of a twist turbine blade," International Journal of Heat and Mass Transfer, vol. 129, pp. 47-58, 2019.

[18] J. Lin, Z. Hu, Z. S. Chen, Y. M. Yang, and H. L. Xu, "Sparse reconstruction of blade tip-timing signals for multi-mode blade vibration monitoring," Mechanical Systems and Signal Processing, vol. 81, pp. 250-258, 2016. 
[19] Z. Ji-wang, Z. Lai-bin, D. Ke-Qin, and D. Li-xiang, "Blade tiptiming technology with multiple reference phases for online monitoring of high-speed blades under variable-speed operation," Measurement Science Review, vol. 18, no. 6, pp. 243250, 2018.

[20] X. Wu and X. Chen, "Internal leakage detection for inlet guide vane system at gas turbine compressor with ensemble empirical mode decomposition," Measurement, vol. 134, pp. 781787, 2019.

[21] Y. Chen, H. Li, L. Hou, J. Wang, and X. Bu, “An intelligent chatter detection method based on EEMD and feature selection with multi-channel vibration signals," Measurement, vol. 127, pp. 356-365, 2018.

[22] X. H. Chen, G. Cheng, X. L. Shan, X. Hu, Q. Guo, and H. G. Liu, "Research of weak fault feature information extraction of planetary gear based on ensemble empirical mode decomposition and adaptive stochastic resonance," Measurement, vol. 73, pp. 55-67, 2015.

[23] S. Zhang, J. Tian, A. Banerjee, and J. Li, “An efficient porcine acoustic signal denoising technique based on EEMD-ICAWTD," International Journal of Rotating Machinery, vol. 2019, Article ID 2858740, 12 pages, 2019.

[24] Y. Zheng, X. Sun, J. Chen, and J. Yue, "Extracting pulse signals in measurement while drilling using optimum denoising methods based on the ensemble empirical mode decomposition," Petroleum Exploration and Development, vol. 39, no. 6, pp. 798-801, 2012. 